\title{
Technical Note: Use of RFLP to Characterize Lactococcus lactis Strains Producing Exopolysaccharides
}

H. Deveau and S. Moineau

Département de Biochimie et de Microbiologie,

Faculté des Sciences et de Génie,

Groupe de Recherche en Écologie Buccale (GREB),

Faculté de Médecine Dentaire

Université Laval,

Québec, Canada, G1K 7P4

\begin{abstract}
Restriction fragment length polymorphism (RFLP) is used to differentiate microorganisms by analysis of their DNA restriction patterns. A modified RFLP procedure is proposed for the rapid characterization of Lactococcus lactis strains producing exopolysaccharides (EPS). The availability of such effective cataloging system is likely to benefit research aimed at identifying lactococcal strains that produce novel EPS.

(Key words: exopolysaccharide, Lactococcus lactis, restriction fragment length polymorphism)
\end{abstract}

Abbreviation key: EPS = exopolysaccharide, $\mathbf{E P S}^{+}=$ ability to produce exopolysaccharides, RFLP = restriction fragment length polymorphism.

Lactococcus lactis is a gram-positive lactic acid bacterium used to acidify milk during the manufacture of several fermented milk products. Most Lactococcus strains contain plasmids that encode industrial phenotypes such as lactose utilization, proteinase activity, phage resistance and, in some cases, production of extracellular polysaccharides (EPS). The production of EPS by these cultures increases the viscosity, decreases the syneresis, and improves the texture of dairy products. Because these polysaccharide-producing strains impart desirable properties, they are increasingly used by the food industry (De Vuyst and Degeest, 1999).

Several lactococcal EPS have already been analyzed for their chemical compositions as well as the location, sequence, and organization of the genes involved in their biosynthesis (Nakajima et al., 1990; Gruter et al., 1992; De Vuyst and Degeest, 1999; van Casteren et al., $2000 \mathrm{a}$ and 2000b). For various purposes (i.e., novel food applications, enhanced EPS production, need of phage-

Received July 26, 2002.

Accepted October 15, 2002.

Corresponding author: S. Moineau; e-mail: Sylvain.Moineau@ bcm.ulaval.ca. unrelated $\mathbf{E P S}^{+}$strains), there is an ongoing search for lactococcal strains producing novel EPS. However, given that the EPS must be extracted, purified, and chemically analyzed, the classical method for characterizing EPS is rather fastidious (Gruter et al., 1992; Cerning, 1995; van Kranenburg et al., 1999). Moreover, Lactococcus strains produce a low quantity of EPS. Thus, the isolation of new wild-type Lactococcus strains producing unique EPS is a daunting task.

Restriction fragment length polymorphism (RFLP) can be used to distinguish strains by the analysis of their DNA restriction patterns. Recently, van Kranenburg et al. (1999) proposed to differentiate lactococcal $\mathrm{EPS}^{+}$strains based on RFLP of the eps operon. The grouping was based on the size of two SstI fragments that hybridized with an eps $\mathrm{B}$ (gene involved in chain length determination) and eps $\mathrm{D}$ (priming glycosyltransferase gene) probes which correlated with the monosaccharide composition of the repetitive unit of the EPS. These analyses led to the grouping of most of the lactococcal strains into three major clusters (van Kranenburg et al., 1999 and Table 1). The polymer produced by strains of group I is made of glucose, galactose, and rhamnose. The EPS synthesized by group II strains contain only galactose. Finally, strains of group III make exopolysaccharides composed of glucose and galactose (van Kranenburg et al., 1999).

Recently, we analyzed the monosaccharide composition of the EPS produced by seven L. lactis strains, which included three reference strains from the study by van Kranenburg et al. (1999), namely MLT3 (group I), H414 (group II), and MLT2 (group III), as well as four strains from our laboratory (SMQ-419, SMQ-420, SMQ-461, SMQ-575). Based on the sugar composition, SMQ-420 and SMQ-575 were classified into group I, SMQ-419 into group III, and SMQ-461 into a fourth and novel group (Deveau et al., 2002). To confirm the grouping of the four $\mathrm{EPS}^{+}$strains from our collection, we used the above RFLP method. Lactococcus lactis strains were grown at $30^{\circ} \mathrm{C}$ in M17 broth supplemented with $0.5 \%$ lactose (LM17) (Quélab, Montréal, Québec, 
Table 1 Sugar composition of the exopolysaccharides produced by seven L.lactis strains and hybridization patterns of digested total DNA.

\begin{tabular}{|c|c|c|c|c|c|c|c|c|}
\hline \multirow[b]{3}{*}{ Group } & \multirow[b]{3}{*}{ Strain } & & & & \multicolumn{4}{|c|}{ Fragment size $(\mathrm{kb})$} \\
\hline & & \multicolumn{3}{|c|}{ Molar ratio ${ }^{1}$} & \multicolumn{2}{|c|}{$S s t \mathrm{I}^{2}$} & \multirow{2}{*}{\multicolumn{2}{|c|}{$\frac{\text { AcyI HindI }}{\begin{array}{c}\text { Probe } \\
\text { eps } \mathrm{D}\end{array}}$}} \\
\hline & & Gal & Glu & Rha & $\begin{array}{l}\text { Probe } \\
\text { eps B }\end{array}$ & $\begin{array}{l}\text { Probe } \\
\text { epsD }\end{array}$ & & \\
\hline & SMQ-419 & 1 & 1,4 & 0,6 & 12 & 0,4 & 9,5 & 3,4 \\
\hline \multirow[t]{2}{*}{ I } & SMQ-575 & 1 & 2,0 & 1,1 & 7,5 & 0,4 & 9,5 & 3,4 \\
\hline & MLT3 & 1 & 1,8 & 0,9 & 7,5 & 0,4 & 9,5 & 3,4 \\
\hline II & H414 & 1 & 0,1 & 0,0 & 7,5 & 15 & 3 & 2,7 \\
\hline \multirow{2}{*}{ III } & SMQ-420 & 1 & 1,6 & 0,0 & 15 & 12 & 7 & 5 \\
\hline & MLT2 & 1 & 1,8 & 0,0 & 6,5 & 12 & 7 & 5 \\
\hline IV & SMQ-461 & 1 & 2,0 & 0,0 & 9 & 13 & 2 & 4,5 \\
\hline
\end{tabular}

${ }^{1}$ Deveau et al. (2002).

${ }^{2}$ van Kranenburg et al. (1999).

Canada). Total DNA of $L$. lactis strains was extracted as described previously (Deveau et al., 2002). Restriction endonucleases (AcyI, HindII, SacI) were used as recommended by the manufacturer (Roche Diagnostics, Laval, Québec, Canada). After restriction, total DNA was electrophoresed on $0.8 \%$ agarose gels in $1 \mathrm{X}$ TAE buffer. Probes were constructed by labeling PCR fragments with the Dig High-Prime labeling kit. The probes were made of PCR products corresponding to the eps $\mathrm{B}$ gene (by using the primers 5'-CGTACGATTCGTACGACCAT-3' and 5'TGACCAGTGACACTTGAAGC-3') and epsD (5'-TGATCCCCGTGTAACGAAGA-3' and 5'-AAGAGAGGCGCTCCCCATAT-3'). Prehybridization, hybridization, washes, and detection by chemiluminescence were performed as suggested by the manufacturer (Roche Diagnostics).

Although the three reference strains (MLT3, H414, MLT2) showed the expected hybridization patterns (van Kranenburg et al., 1999), three of the four lactococcal strains from our laboratory could not be classified by RFLP (Figure 1, Table 1). Two disadvantages were found with the proposed RFLP method. First, the SacI (isoschizomer of $S s t \mathrm{I}$ ) fragments that hybridized with the eps $\mathrm{B}$ and eps $\mathrm{D}$ probes were often too large $(>12$ $\mathrm{kb}$ ) for accurate size estimation in Southern analysis (Figure 1). But most importantly, a critical SacI site is located upstream (outside) the eps operon (the other SacI site is within the operon), indicating that the DNA sequence flanking the operon will dictate the size of the hybridizing fragment rather than the eps operon (Figure 2). Thus, the hybridization signals are reflecting the variability of the nucleotide sequence upstream of the operon rather than the variability of the operon itself. These findings led to the modification of the classification procedure.
A

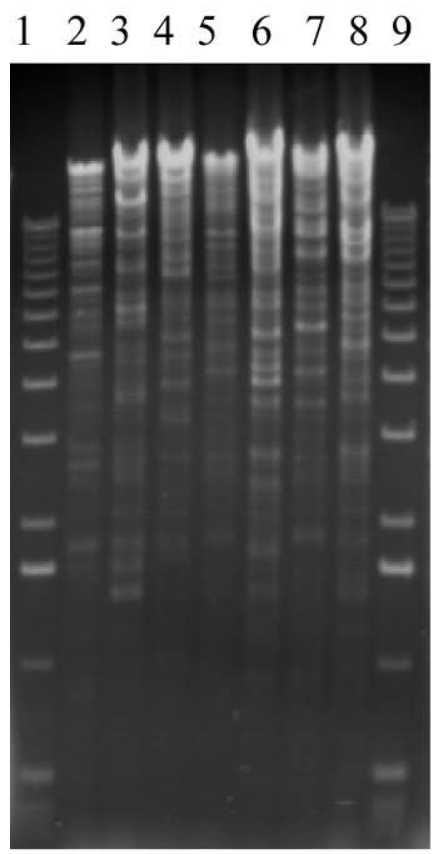

B
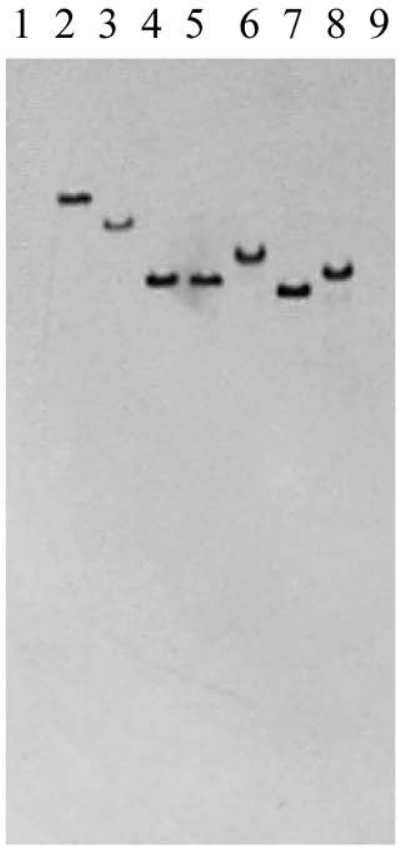

C
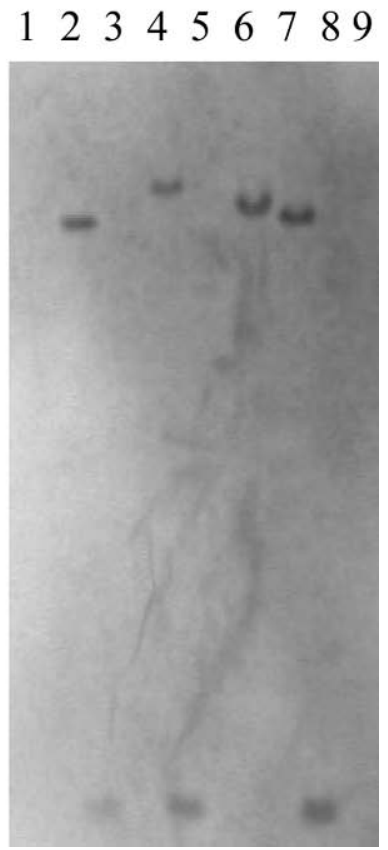

Figure 1. Differentiation of Lactococcus lactis eps gene clusters by the restriction fragment length polymorphism method of van Kranenburg et al. (1999). Lanes 1 and 9, 1-kb ladder (Gibco/BRL); lane 2, SMQ-420; lane 3, SMQ-419; lane 4, H414; lane 5, SMQ-575; lane 6, SMQ461; lane 7, MLT2; lane 8, MLT3. Panel A: Total DNA digested SacI; Panel B: Hybridization with epsB probe; Panel C: Hybridization with epsD probe. 

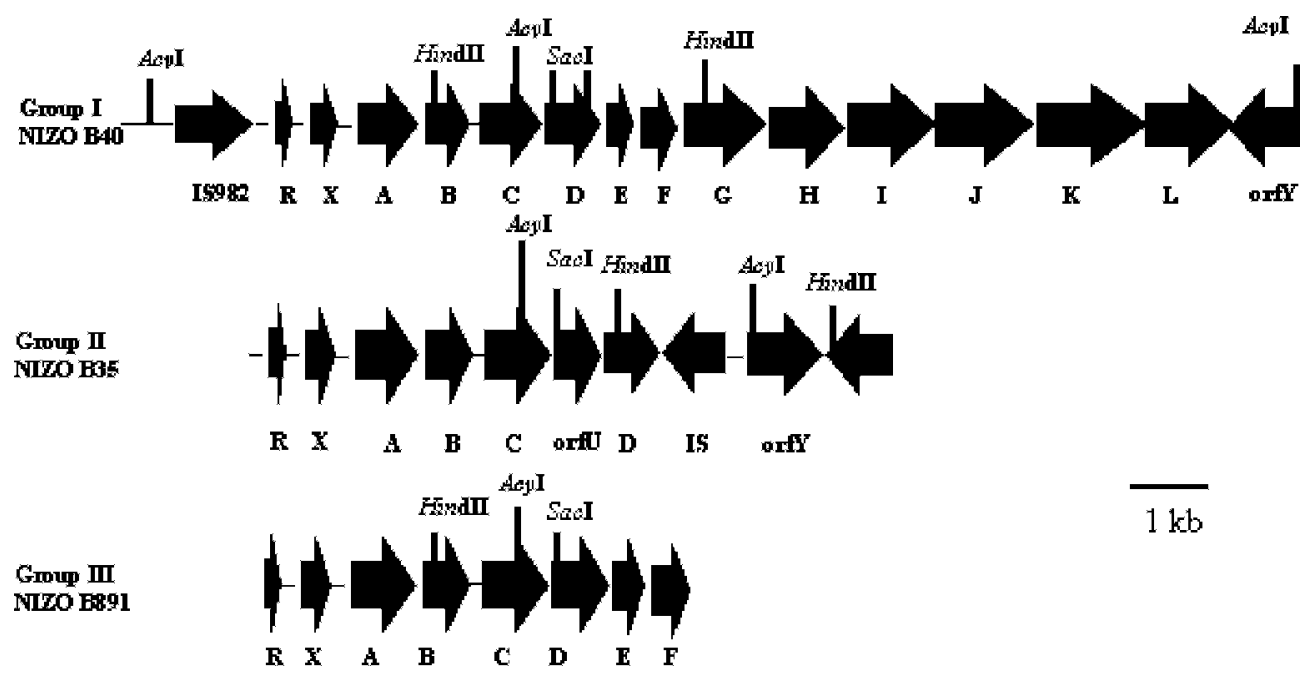

Figure 2. Localization of SacI, AcyI, and HindII restriction sites in the eps gene cluster (Accession number: group 1, U93364; group II, AF100297; group III, AF100298).

The total DNA of the seven strains was digested with HindII and AcyI endonucleases. These restriction enzymes were selected based on nucleotide sequences of lactococcal eps operon available in databases that indicated the presence of HindII and AcyI sites within the eps operon of the three groups (Figure 2). The restriction fragments were then separated by electrophoresis and transferred onto a membrane. The eps $\mathrm{D}$ probe was used for the hybridization because it is the most conserved gene within the eps operon of the three groups. The hybridization results are presented in Figure 2 and summarized in Table 1 . The reference strain MLT3 (group I) had a 9.5-kb AcyI fragment as well as a $3.5-\mathrm{kb}$ HindII fragment that hybridized with the eps D probe (Figure 3, panels A and B, lane 8). Identical results were obtained with the strains SMQ-419 and SMQ-575 (Figure 3, panels A and B, lanes 3 and 5, respectively). The strain H414 (group II) had unique hybridization patterns with a $3.0-\mathrm{kb} A c y \mathrm{I}$ and a $2.7-\mathrm{kb}$ HindII fragments that hybridized with the epsD probe (Figure 3, panels A and B, lane 4). The reference strain MLT2 (group III) and SMQ-420 had identical hybridization signals with the eps $\mathrm{D}$ probe, which included a 7.0$\mathrm{kb}$ Acy I fragment and a 5.0-kb HindII fragment (Figure 3 , panels A and B, lanes 2 and 7, respectively). Finally, the strain SMQ-461 also had unique hybridization patterns with a 2.0-kb AcyI and a 4.7-kb HindII fragments that hybridized with the eps D probe (Figure 3, panels A and B, lane 6), confirming that it belongs to a fourth and novel group (Deveau et al., 2002). The classification of the seven $\mathrm{EPS}^{+}$L. lactis strains by this new RFLP procedure is in agreement with the previous analysis of the monosaccharide composition of the EPS (Deveau
A

$\begin{array}{lllllllll}1 & 2 & 3 & 4 & 5 & 6 & 7 & 8 & 9\end{array}$

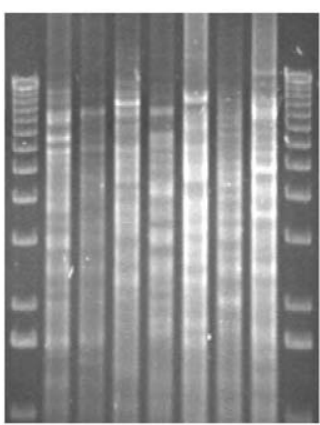

12234556789

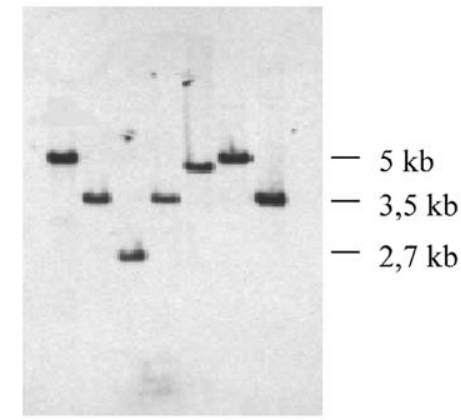

B

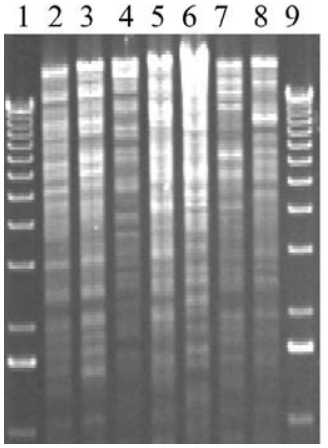

D

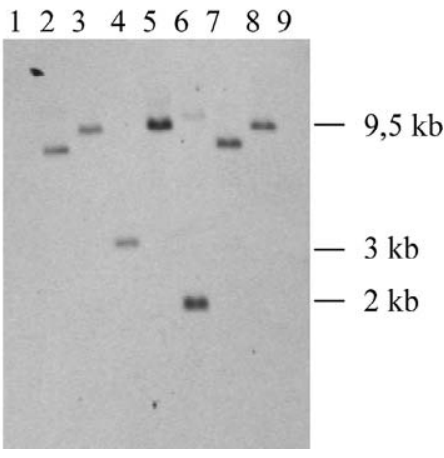

Figure 3. Differentiation of Lactococcus lactis eps gene clusters by the modified restriction fragment length polymorphism method. Lanes 1 and 9, 1-kb ladder (Gibco/BRL); lane 2, SMQ-420; lane 3, SMQ-419; lane 4, H414; lane 5, SMQ-575; lane 6, SMQ-461; lane 7, MLT2; lane 8, MLT3. Panel A: Total DNA digested HindII; Panel B: Total DNA digested AcyI; Panels C and D: Hybridization with epsD probe. 
et al., 2002). Moreover, the eps operon of SMQ-575 was recently sequenced, and it is $100 \%$ identical at the nucleotide level to the eps operon of the strain NIZO B40 (GenBank accession number AF036485), which is also a member of group I, confirming the classification of SMQ-575 into this group (H. Deveau and S. Moineau, unpublished).

Thus, we propose a modified RFLP procedure using HindII and AcyI rather than SacI for rapid differentiation of EPS-producing lactococcal strains. It is worth mentioning that additional strains should be tested to further validate the method. Notwithstanding, this cataloging system should speed up the isolation of lactococcal strains that produce novel EPS as well as the isolation of phage-unrelated strains producing identical EPS. As novel lactococcal EPS are characterized, this RFLP method is also likely to be refined accordingly.

\section{ACKNOWLEDGMENTS}

We are grateful to $R$. van Kranenburg for providing the strains MLT2 and MLT3. We thank D. Roy and M.-R. Van Calsteren for their helpful discussions. The authors wish to thank the Natural Sciences and Engineering Research Council of Canada (Research Partnerships Program Research Network on Lactic Acid Bacteria), Agriculture and Agri-Food Canada, Novalait,
Inc., Dairy Farmers of Canada, and Institut RosellLallemand, Inc. for financial support. H. D. is a recipient of a FCAR graduate scholarship.

\section{REFERENCES}

Cerning, J. 1995. Production of exopolysaccharides by lactic acid bacteria and dairy propionibacteria. Lait 75:463-472.

Deveau, H., M.-R. Van Calsteren, and S. Moineau. 2002. The effect of exopolysaccharides on phage-host interactions in Lactococcus lactis. Appl. Environ. Microbiol. 68:4364-4369.

De Vuyst, L., and B. Degeest. 1999. Heteropolysaccharides from lactic acid bacteria. FEMS Microbiol. Rev. 23:153-177.

Gruter, M., B. R. Leeflang, J. Kuiper, J. P. Kamerling, and J. F. G. Vliegenthart. 1992. Structure of the exopolysaccharide produced by Lactococcus lactis subspecies cremoris H414 grown in a defined medium or skimmed milk. Carbohydr. Res. 231:273-291.

Nakajima, H., S. Toyoda, T. Toba, T. Itoh, T. Mukai, H. Kitazawa, and S. Adachi. 1990. A novel phosphopolysaccharide from slimeforming Lactococcus lactis subsp. cremoris SBT 0495. J. Dairy. Sci. 73:1472-1477.

van Casteren, W.H.M., P. de Waard, C. Dijkema, H. A. Schols, and A.G.J. Voragen. 2000a. Structural characterisation and enzymatic modification of the exopolysaccharide produced by Lactococcus lactis subsp. cremoris B891. Carbohydr. Res. 327:411-422.

van Casteren, W.H.M., C. Dijkema, H. A. Schols, G. Beldman, and A.G.J. Voragen. 2000b. Structural characterisation and enzymatic modification of the exopolysaccharide produced by Lactococcus lactis subsp. cremoris B39. Carbohydr. Res. 324:170-181.

van Kranenburg, R., H. R. Vos, I. I. van Swam, M. Kleerebezem, and W. M. de Vos. 1999. Functional analysis of glycosyltransferase genes from Lactococcus lactis and other Gram-positive cocci: Complementation, expression, and diversity. J. Bacteriol. 181:6347-6353. 